EPJ manuscript No.

(will be inserted by the editor)

\title{
Polyelectrolyte multilayer formation: electrostatics and short-range interactions
}

\author{
Adi Shafir ${ }^{a}$ and David Andelman ${ }^{b}$ \\ School of Physics and Astronomy, Raymond and Beverly Sackler Faculty of Exact Sciences, Tel Aviv University, Ramat Aviv, \\ Tel Aviv 69978, Israel
}

04 December 2005

\begin{abstract}
We investigate the phenomenon of multilayer formation via layer-by-layer deposition of alternating charged polyelectrolytes. Using mean-field theory, we find that a strong short-range attraction between the two types of polymer chains is essential for the formation of multilayers. For strong enough short-range attraction, the adsorbed amount per layer increases (after an initial decrease), and finally it stabilizes in the form of a polyelectrolyte multilayer that can be repeated hundreds of times. For weak short-range attraction between any two adjacent layers, the adsorbed amount (per added layer) decays as the distance from the surface increases, until the chains stop adsorbing altogether. The dependence of the threshold value of the short-range attraction as function of the polymer charge fraction and salt concentration is calculated.
\end{abstract}

PACS. 82.35.Gh Polymers on surfaces; adhesion - 82.35.Rs Polyelectrolytes - 61.41.+e Polymers, elastomers, and plastics

\section{Introduction}

The study of polyelectrolyte (PE) chains interacting with charged surfaces has generated a great deal of attention in recent years. This interest arises, in part, because of the numerous biological and industrial applications. The adsorption and depletion of polyelectrolytes on charged surfaces have been extensively studied using analytical [1,2, [3, 4,5, 6 and numerical [7, 8,9, 10,11] solutions of the nonlinear mean-field equations, scaling considerations 7,8 , ' 9, 10, 11 12 13, 14 15 16, 17, multi-Stern layers of discrete lattice models [18, 19,20, 21] and computer simulations [22. 23 24. 25.

In recent years, formation of polyelectrolyte multilay' ers has been investigated experimentally $[26 \mid 27,28,29,30$,

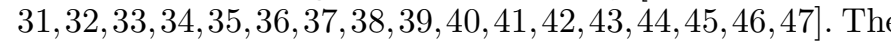
multilayers are composed of alternating positively and negatively charged PEs and are constructed via a layer-bylayer adsorption of polyelectrolyte chains, shown schematically in Fig. 1. The first stage is to dip a charged surface into a solution of oppositely charged polyelectrolytes and salt. After the polyelectrolyte chains adsorb on the charged surface, the surface is taken out of the solution and washed in a clear water solution. The washed surface and adsorbed layer are then placed in a solution of another polyelectrolyte, of an opposite charge to the first PE chain (see Fig. 1), and then washed again. This pro-

\footnotetext{
a e-mail: shafira@post.tau.ac.il

b e-mail: andelman@post.tau.ac.il
}

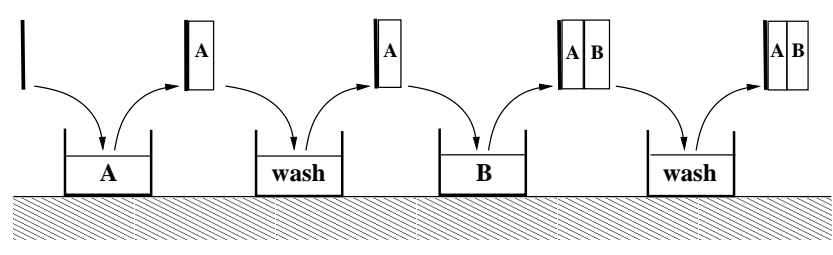

Fig. 1. A schematic illustration of the layer-by-layer PE deposition process. The charged surface is first dipped into a solution of oppositely charged PE chains (marked 'A'). After the chains adsorb onto the charged surface, the surface is removed and dipped into a clear water solution (marked 'wash'), removing any extra non-adsorbed chains. The surface is then dipped into another solution of PEs (marked 'B'), carrying a charge Sopposite to the previously adsorbed PE chains, and washed again ('wash'). The process of dipping and washing: 'A', wash, 'B', wash, 'A', wash,... can then be repeated for several hundred layers.

cess can be repeated for several hundred times and results in a PE multilayer build-up 32. More recent studies 30 . 31 have shown that these multilayers have interesting and potentially useful applications both for planar and spherical geometries, leading the way to creation of multilayered and hollow spherical capsules.

Theoretical models for multilayer formation have also been considered in recent years 6, 11, 48, 49, 50,51,52. In 
previous studies 6. 68 , it was suggested that the inversion of the surface charge by polyelectrolyte adsorption occurs under the following conditions: (i) sufficiently large salt concentration; (ii) theta solvent conditions; and, (iii) weak short-range (SR) interactions between the surface and the $\mathrm{PE}$ chains. The charge inversion, together with complexation of polyanions and polycations have been used to model the multilayer formation [48.

In a previous publication [1], we reached different conclusions by solving numerically the relevant mean-field equations for the PE adsorption. We found that surface charge inversion will not occur under a broad range of system parameters. More specifically, it was shown that full charge inversion occurs only for strong enough SR surface$\mathrm{PE}$ interactions. Related results have been reported in a separate study [51, where the full charge inversion was found to occur only for strongly hydrophobic PE-backbone. Namely, PE chains in poor solvent conditions. The strong hydrophobicity in the bulk creates an effective attraction to the surface, replacing the bare surface-PE short-range attraction.

In the present work, we study the formation of alternating charged PE multilayers as a function of polyelectrolyte charge, added salt and SR interaction between the $\mathrm{PE}$ chains. We find that strong SR (non-electrostatic in origin) interactions are necessary for the formation of such multilayers, and that the adsorbed charge of the alternating layers is not necessarily equal, or even close to, the initial surface charge. Our analysis shows that the adsorbed amount (per added layer) in the initially adsorbed layers always decreases. If the SR interactions between the PE chains are too small to attract another PE layer, the multilayer formation will stop after a small number of layers. However, if the SR attraction between the alternating $\mathrm{PE}$ layers is significant, the adsorbed amount (per added layer) starts to increase back, and then saturates and forms a stable multilayer stack. We also show how the multilayer formation depends on the solution salinity and PE charge fraction.

In the next section, Sec. 2] we present the mean field equations for multilayer formation and the numerical method used to solve them. The numerical results for multilayer formation follow in Sec. 3] and their discussion in Sec. 4. We end with conclusions and suggestions for future research in Sec. 5

\section{The Mean Field Equations}

Consider an aqueous solution in contact with a bulk reservoir of salt ions, and a dilute bulk concentration of long polyelectrolyte chains. The solution is in contact with an infinite and planar surface. The surface is oppositely charged and attracts the PE chains. The mean-field equations for this system were formulated in Refs. [7, 8, 9 , 10], and are repeated here.

$$
\frac{\mathrm{d}^{2} \zeta}{\mathrm{d} x^{2}}=\kappa^{2} \sinh \zeta-4 \pi l_{B} f \phi^{2}
$$

$$
\frac{a^{2}}{6} \frac{\mathrm{d}^{2} \phi}{\mathrm{d} x^{2}}=v \phi^{3}+f \zeta \phi+\omega^{2} \phi^{5}
$$

$\phi^{2}$ is the local monomer concentration, $x$ the distance from the charged surface, $\zeta=e \psi / k_{B} T$ the renormalized (dimensionless) electrostatic potential, $a$ the monomer size, and $f$ the charge fraction of the PE monomers. The DebyeHückel length $\kappa^{-1} \equiv\left(8 \pi l_{B} c_{\text {salt }}\right)^{-1 / 2}$ is the screening length for electrostatic interactions in presence of salt ions, and $l_{B} \equiv e^{2} / \varepsilon k_{B} T$ is the Bjerrum length, which is approximately $7 \AA$ for water with $\varepsilon=80$ and at room temperature.

Equation (1) is the Poisson-Boltzmann equation, where the salt ions obey the Boltzmann distribution and together with the monomer charges they act as charge sources for the electrostatic potential. Equation (2) is the Edwards equation for the monomer order parameter $\phi$, where the chains are subject to an external potential composed of an electrostatic potential and an excluded volume interaction between the monomers. Note that in Eq. (2) we included both the second virial term modeled by $v$ and the third virial one modeled by $\omega^{2}$. In most previous studies of $\mathrm{PE}$ adsorption the third virial term has been omitted, because of the dominance of the electrostatic interactions and the second virial term. In the case of multilayer formation, however, the third virial term becomes significant, as is explained in the next section. Finally, we note that Eqs. (11) and (2) are written for vanishingly small bulk concentration of monomers, and under the assumption that the ground state dominance approximation holds.

In order to model the build-up of multilayers, we note that the experimental multilayer build-up is done via a layer-by-layer adsorption of cationic and anionic PE chains, as is illustrated in Fig. 1 and explained in the introduction. During the adsorption of each layer, the chains from previously adsorbed layers are believed not to dissolve back into the solution. Note that the layer-by-layer build-up is not a thermodynamically equilibrium process. Any modeling of this phenomenon should take into account these specific stages.

In our model, electrostatic and short-range (SR) interactions with the PE chains of the previous layers are taken into account. The SR interactions between the anionic and cationic PEs may have several origins. The repulsive SR interactions include excluded volume interactions, while an attractive SR interaction (beside the electrostatic attraction) arises from polyelectrolyte complexation. We do not offer in the present work a detailed explanation for the complexation origin. We rather assume its existence, which yields an effective SR attractive interaction, and investigate under which conditions it will lead to multilayer formation. We assume that the electrostatic attraction and ion pairing between the chains in the adsorbed multilayer and the adsorbing PE chains allow the adsorbing chains to penetrate the multilayer. This penetration slows down the PE chains dissolution back into the solution, and creates an effective SR attraction. This interaction has a non-equilibrium origin, but for dense layers, it should last long enough to allow for multilayer formation. 
The electrostatic interaction between the PE chains is taken into account by adding the PE chain charges in the Poisson-Boltzmann treatment, Eq. (11). The SR interactions between the cationic and anionic PE chains are taken into account very simply by adding a different interaction parameter $\chi \neq v$ in Eq. (4). Hereafter, we assume that the charged fraction $f$ of monomers for the negatively and positively charged PE chains is the same. As the PE adsorption is done layer by layer, the equations governing the adsorption of the $i$ th layer are:

$$
\begin{gathered}
\frac{\mathrm{d}^{2} \zeta}{\mathrm{d} x^{2}}=\kappa^{2} \sinh \zeta-4 \pi l_{B} f\left(z_{i} S_{s}+z_{i+1} S_{o}\right) \\
\frac{a^{2}}{6} \frac{\mathrm{d}^{2} \phi_{i}}{\mathrm{~d} x^{2}}=v S_{s} \phi_{i}+f z_{i} \zeta \phi_{i}-\chi S_{o} \phi_{i}+\omega^{2} S_{s}^{2} \phi_{i}
\end{gathered}
$$

where $\zeta(x)$ is the electrostatic potential, and $\phi_{i}^{2}$ and $f z_{i}$ denote the monomer concentration and monomer valency of the $i$ th layer, respectively. The two above equations are solved iteratively for the $i$ th layer concentration $\phi_{i}$, while assuming that monomer concentrations from all previously adsorbed layers, $i-1, i-2, \ldots, 1$ are fixed and known from previous iterations. These SR interactions are contained in the two sums appearing in the right hand side of Eqs. (3) and (4), $S_{s}$ and $S_{o}$. The sum

$$
S_{s} \equiv \sum_{j=i, i-2 \ldots} \phi_{j}^{2}(x)
$$

is the monomer concentration at the point $x$, summed over all similarly charged layers: $j=i, i-2, \ldots$, which repel the monomers of the $i$ th layer. Similarly, the other sum:

$$
S_{o} \equiv \sum_{j=i-1, i-3 \ldots} \phi_{j}^{2}(x)
$$

is summed over all oppositely charged layers having an attractive SR interaction with the newly adsorbing PEs. It should be noted that $S_{s}$ and $S_{o}$ are both functions of the layer number $i$, but the subscript $i$ is omitted for simplicity. In the following, we consider only monovalent PEs and set the odd layers as negatively charged, $z_{2 i+1}=-1$, whereas the even ones as positively charged, $z_{2 i}=1$. We also note that for high monomer concentrations the terms $S_{0}$ and $S_{s}$ are large, and thus higher orders of both the excluded volume and the attractive interactions may be necessary. However, in this simple model we restrict ourselves to the third order only.

The solution of the pair of 2nd order differential equations, Eqs. (3) and (4), requires four boundary conditions. Two of them are for the bulk where we choose $\phi_{i}(x \rightarrow \infty)=0$, corresponding to a negligible amount of $\mathrm{PE}$ in the bulk solution (dilute solution), and zero value for the electrostatic potential, $\zeta(x \rightarrow \infty)=0$. At the solid surface, $x=0$, we use the electrostatic boundary condition $\mathrm{d} \zeta /\left.\mathrm{d} x\right|_{x=0}=-4 \pi l_{B} \sigma$, where $\sigma$ is the surface charge density. For the first PE layer that adsorbs directly onto the solid surface $(i=1)$, we impose the Cahn-de Gennes attractive boundary condition $[6,53] \mathrm{d} \ln (\phi) /\left.\mathrm{d} x\right|_{x=0}=-d^{-1}$, where $d$ is a characteristic length for the SR interactions between the surface and the PE chains, and $d>0$ corresponds to an attractive surface.

For all subsequent layers, $i \geq 2$, we expect the PE chains to partially penetrate into the previous layers because of the complexation. In order to avoid the possibility of fully interpenetrated layers, we introduce a hard wall for each layer at an arbitrary location $x_{i}^{*}$. Otherwise, in our case the cationic and anionic polyelectrolytes would form a neutral complex, which will not be able to form a stable PE multilayer. Because it is known from experiments that the adsorbing $\mathrm{PE}$ chains of any specific layer do not fully mix with the previous layers we introduce the concept of the hard wall. Its justification would require further studies. For the $i$ th deposited layer, there is a (artificial) hard wall $x_{i}^{*}$ inside the previous layers so that no monomers from the $i$ th layer can reach the region $x<x_{i}^{*}$. In order to simplify notation, the layer index $i$ is dropped from the hard wall notation, $x^{*}$.

The adsorption of every layer brings about a reversing of the overall charge of the surface-PE-small ion complex. When the adsorbed amount of ions and PE chains exactly balances the total charge of the surface and previous adsorbed layers, the electrostatic field perpendicular to the surface is exactly zero (Gauss law). The hard wall of the adsorbing PE layer $x^{*}$ is taken somewhat arbitrarily as the point where the electric field is zero. As an example, the location of $x^{*}$ is depicted in Fig. 2.

Our choice of $x^{*}$ is motivated by our understanding the complexation procedure. The driving forces for the adsorbing PEs to penetrate the preceding layer are the electrostatic attraction between oppositely charged PE chains and the ion pairing between charged monomers. This electrostatic attraction is driven by an attractive electric field for $x>x^{*}$. For $x<x^{*}$, the electrostatic field repels the adsorbing PE, and no significant complexation in that region is expected. Since we assume that the SR attraction is a result of non-equilibrium complexation between electrostatically attracted PE chains, we do not take $x^{*}$ to be dependent on $\chi$. It is important to note that such complexation cannot occur between similarly charged PE chains, because the repulsive interaction between the two chains as well as the excluded volume repulsion would drive the chains to separate rather than inter-penetrate.

The numerical procedure used to solve Eqs. (1) and (2), as applied to a single adsorbing PE layer, is based on the relaxation method [54, and was presented in detail in a previous publication 10. Here, we use the same procedure for the layer-by-layer build-up. After obtaining the solution for the first PE layer concentration, $\phi_{1}^{2}(x)$, and its resulting potential, $\zeta(x)$, the layer monomer concentration profile $\phi_{1}^{2}(x)$ is frozen and added as a charge density source to the right-hand-side of Eqs. (34). These equations are now solved for the second layer using the hard wall $x^{*}$ and the two virial coefficients, $v$ and $\chi$. The procedure is then repeated iteratively for all following layers in order to obtain a multilayer stack. 


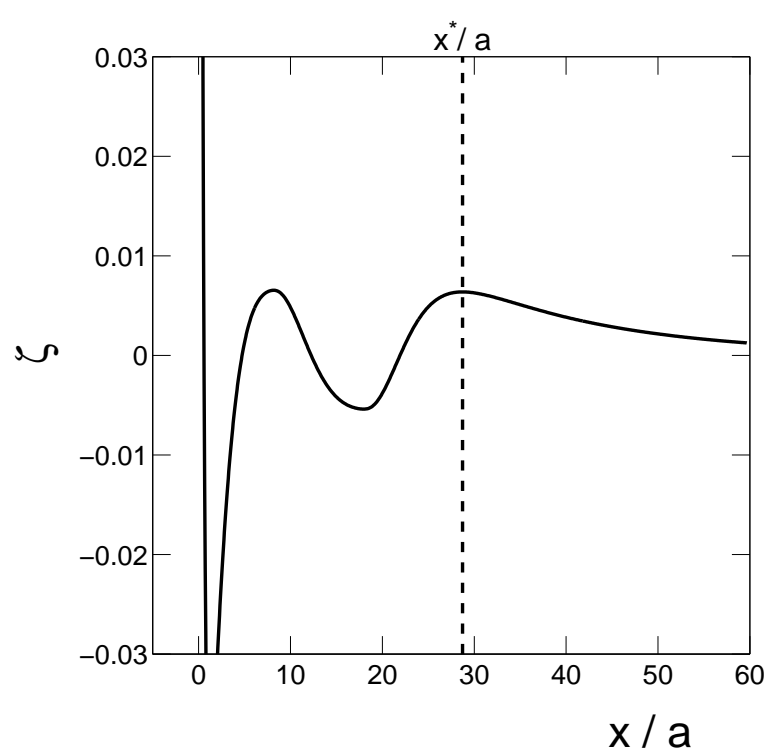

Fig. 2. The electrostatic potential $\zeta$ (in dimensionless units) of the first four alternating PE layers is presented as a function of the distance from the surface $x$. In each extremum point, the electrostatic field $\mathrm{d} \zeta / \mathrm{d} x$ changes sign, allowing the multilayer to attract an oppositely charged polyelectrolyte. The location of the farthest peak (marked by a dashed line) is taken as $x_{5}^{*}=x^{*}$ - the hard wall condition for the interpenetration of the next (fifth) layer. This electrostatic potential was taken from the numerical profiles such as in Fig. 3. All parameter values are specified in Fig. 3. We note that the layers shown here are the initially adsorbed layers, and hence the strength of the potential oscillations is strongly affected by the existence of a charged wall. The amplitude of the electrostatic potential oscillations in the more distal region increases towards the final layers, due to the increase in the adsorbed layer amount and charge.

\section{Results}

Our calculations show a strong dependence of the multilayer formation on the value of the SR attraction coefficient $\chi$ in the case of a weakly good solvent, modeled via the $2^{\text {nd }}$ virial coefficient $v=0.05 a^{3}$ where $a$ is the monomer size. For low amounts of added salt $c_{\text {salt }}=0.1 \mathrm{M}$, the formation of multilayers requires very large $\chi / a^{3} \sim 3$ values, while for higher amounts of salt $c_{\text {salt }}=1 \mathrm{M}$ the required $\chi$ values drop to more realistic values of $\chi / a^{3} \sim$ $0.4-1$. For all salt concentrations, low $\chi$ values cause the adsorbed amount of monomers in each layer to decay strongly with the layer number, so that very few layers are formed. For high $\chi$ values, the amount of adsorbed monomers decreases for the initial layers and then increases back. The adsorbed amount in each layer is found to reach a stable value because of the third virial term. The threshold $\chi$ value is shown below to depend on the amount of salt in the solution as well as the initial surface charge and the monomer charged fraction.

The numerical solution of Eqs. (3) and (4) yields the formation of multilayers, as presented in Fig. 3, under the proper choice of parameters. As can be seen from the fig-

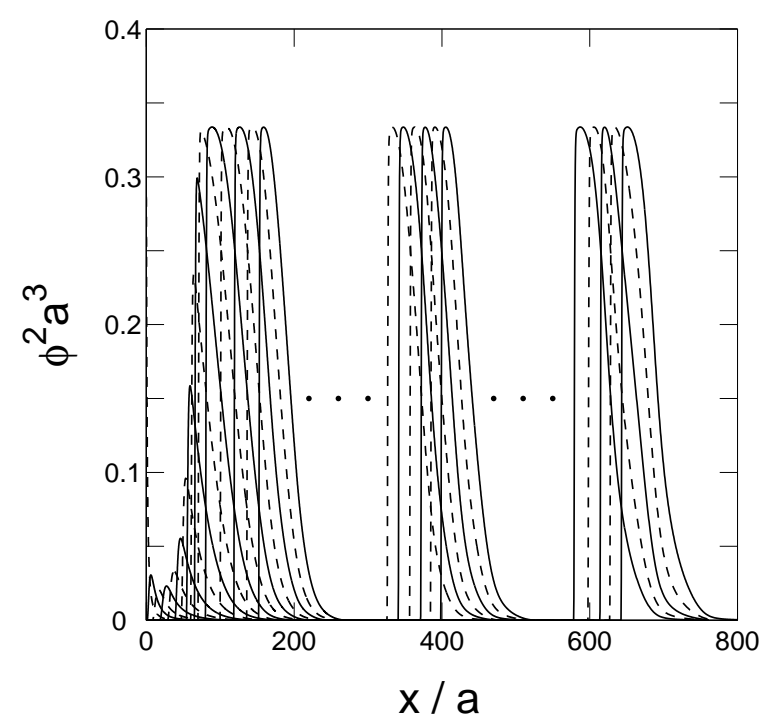

Fig. 3. The formation of a multilayer stack is shown for high $\chi$ value, $\chi=0.55 a^{3}$, and the choice of parameters: $c_{\text {salt }}=1.0 \mathrm{M}$, $a=10 \AA, \sigma=2 \cdot 10^{-3} \AA^{-2}, d=50 \AA$. For clarity purpose, we show only three groups of layers: layers 1-16 (left), 29-33 (middle) and 46-50 (right). For both polymers $v=0.05 a^{3}$, $\omega^{2}=0.5 a^{6}, f=0.5$. The aqueous solution has $\varepsilon=80$ and $T=300 \mathrm{~K}$. The polycation profiles are marked by a solid line, while those of the polyanions by a dashed line. Three regions can be seen in the graph. Near the surface the multilayer concentration decays rapidly for layers 1-5 (proximity region), and then increases rapidly in layers 6-10 (intermediate region). The third region is where the multilayer concentration stabilizes. This stabilization occurs at a higher value than in the initially adsorbed layers. The layers in the distal region are highly interpenetrating, so that any layer interacts with about five other layers during its adsorption process. Note that the lowest monomer volume fraction in the proximity region is 0.025 , which is much lower than in the distal region. However, this layer is strongly complexated with the previous layers and should still be dense enough to survive the washing procedure.

ure, the multilayer can be divided into three spatial regions. In the proximity region, containing the first few layers, the adsorbed amount decreases substantially. In the intermediate region (layers 6-10), the monomer concentration increases rapidly to much higher values. In the distal region, (under some conditions discussed below) the adsorbed amount stabilizes, and the multilayer formation continues. The adsorbed layers are shown to be very wide (of the order of tens of nanometers) and highly concentrated. The interpenetration between the layers looks to be quite significant. The location $x^{*}$, where the next layer begins to adsorb, is shared by monomers from all four previous layers. This strong interpenetration is the driving force of the multilayer formation, since it allows for a strong interlayer SR attraction. Without it no significant overcharging is achieved. The overall charge of each adsorbed layer is much higher than the initial surface charge, showing that there is no exact charge reversal in PE adsorption. 


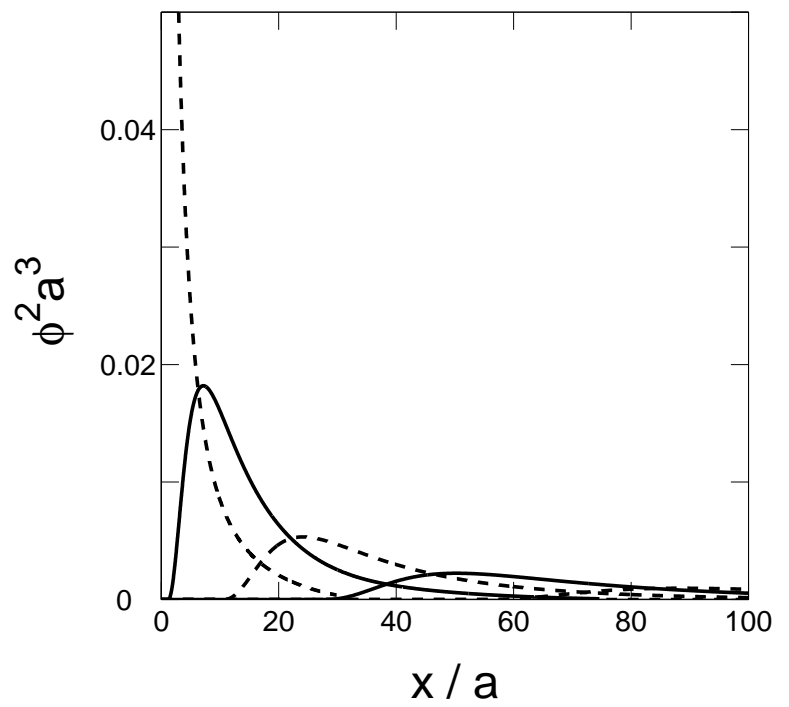

Fig. 4. The multilayer profile with lower $\chi$ value, $\chi=0.39 a^{3}$, and all other parameters as in Fig. 3. For this low $\chi$, the decrease in the non-electrostatic interaction between the PE chains causes the adsorbed layers to decay rapidly after four layers, and no stable multilayer stack is formed.

The multilayer formation is characteristic of high $\chi$ values. In the opposite limit of low $\chi$ values, no stable multilayer stack is formed because the complexation between the layers is not strong enough. This case is shown in Fig. 4, which is obtained for similar parameters as Fig. 3 except for a lower SR interaction coefficient $\chi$. The figure shows that the adsorbed amount in each subsequential layer decays rapidly, until an additional layer cannot be adsorbed, and the formation of a stable multilayer stack is not possible.

Within our model the formation of a stable multilayered stack requires a third order virial term. Only when a strong enough third-virial coefficient, of order $\omega^{2} \sim 0.5 a^{6}$, is added to the SR interaction term, the multilayer concentration stabilizes at high, but still physical, values. When the third virial coefficient is too low, the adsorbed layer concentration does not saturate, and rather reaches unrealistic high values. Our calculations also show that an increase in the second virial coefficient is not enough to stabilize the multilayers. It just drives up the threshold value of $\chi$. The spatial region where the adsorbed amount stabilizes is the multilayer distal region, and it can be continued for as many as 80 layers (in our calculations) without any noticeable decay in the adsorbed amount in each layer.

We end this section by showing three further results. In Fig. 5 we show the overall thickness of the adsorbed layers from Fig. 3 as a function of layer number. In the mean-field model, the thickness of the adsorbed layer is taken as the position of the last monomer concentration peak, which is a lower estimate for the layer width. The adsorbed layer width increases weakly for the first few layers (proximity and intermediate regions), and then increases almost linearly with the layer number (distal region) for the entire 50 layers. The linear increase shows that the

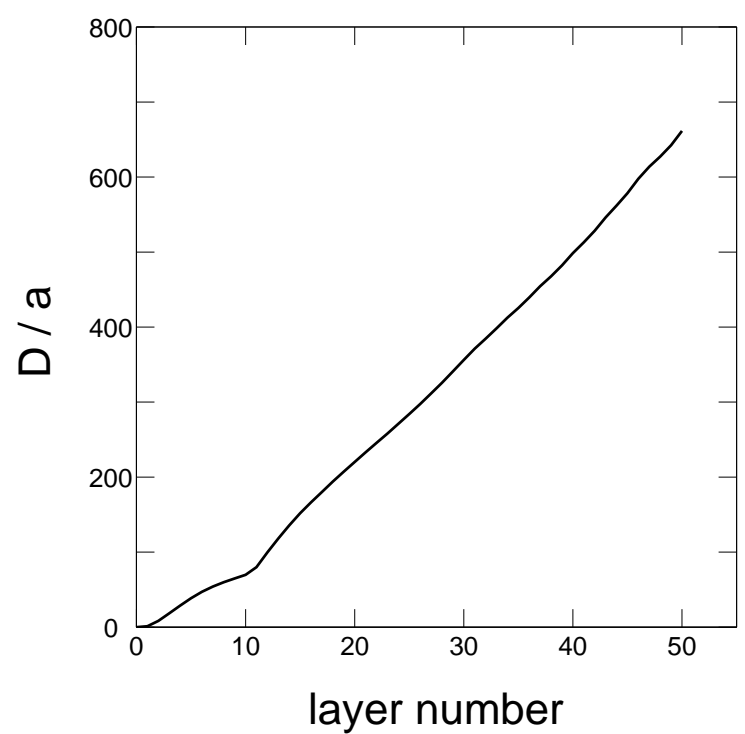

Fig. 5. The total width $D$ of the adsorbed multilayer in Fig. 3 is plotted as a function of the layer number. The (incremental) layer width is extracted from the peak position in the monomer concentration. The total width $D$ is seen to increase mildly for the initially adsorbed layers (proximity and intermediate regions), and then increase almost linearly, corresponding to the stable multilayer formation, with a constant thickness per each adsorbed layer.

multilayers are indeed stable and reaches very high layer numbers.

In Fig. 6 we present the threshold strength of $\chi$ that is needed to form multilayers as a function of the added salt amount. As can be seen from this graph, an increase in the amount of added salt causes the necessary $\chi$ to decrease strongly for low salt concentrations. For higher salt concentrations the $\chi$ value is almost constant. The dependence of $\chi$ on $c_{\text {salt }}, \chi \sim c_{\text {salt }}^{\alpha}$ fits roughly a power law with $\alpha \simeq-0.8$, as can be seen in the inset of Fig. 6 . However, this empirical scaling is valid only for small range of salt concentrations.

The dependence of the threshold $\chi$ value on the monomer charged fraction $f$ is presented in Fig. 7. The $\chi$ threshold increases with the increase of $f$. Here, too, the numerical results do not imply any simple scaling relation between $f$ and $\chi$ (see inset of Fig. 7).

\section{Discussion}

The spatial behavior in Figs. 3 and 4 can be explained by the following argument. In the proximity region, close to the surface, the adsorbed layers have high monomer concentration and small width. This small width does not allow for significant complexation between the adjacent layers. Therefore, it causes the SR attraction between the already adsorbed layers and the adsorbing PE chains of the current layer to be low. This, in turn, causes a decrease in the concentration of adsorbing monomers, accompanied by an increase in the layer width. 


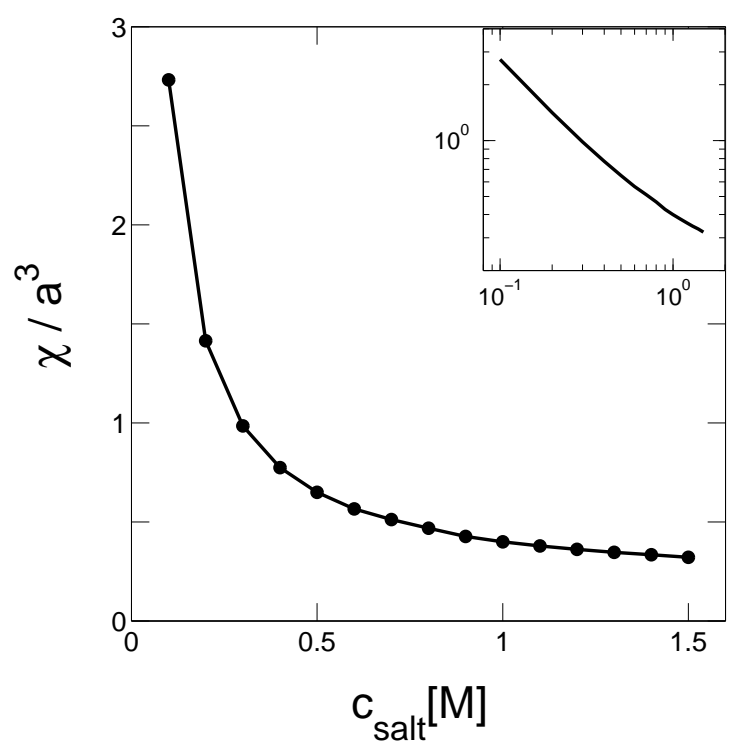

Fig. 6. The threshold value of $\chi$ needed to create stable multilayers is plotted as a function of $c_{\text {salt }}$. For low salt we get high values of $\chi \sim 3 a^{3}$, while for high salt the value of $\chi$ has lower values, around $0.5 a^{3}$. All other parameters are as in Fig. 3, except $d=10 \AA$. The inset shows the same dependence on a $\log -\log$ plot. For low $c_{\text {salt }}$ values, the slope of the line can be fit to $\chi \sim c_{\text {salt }}^{-0.8}$, but for higher salt concentrations the exponent becomes lower. Since these changes occur over a single decade in $c_{\text {salt }}$ values, there does not appear to be a good scaling law for $\chi$ as function of $c_{\text {salt }}$.

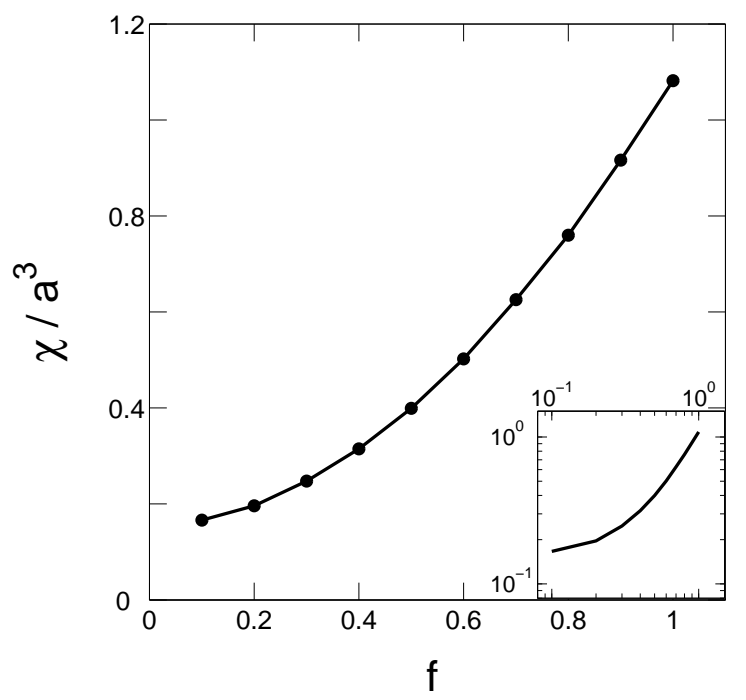

Fig. 7. The threshold value of $\chi$ needed to create stable multilayers is plotted as a function of $f$. For low $f$ values, the monomer-monomer repulsion is low, and only a weak shortrange attraction between the polymers is needed. For higher $f$ values the threshold value of $\chi$ increases. All other parameters are as in Fig. 3, except $d=10 \AA$. The inset shows the same dependence on a log-log plot. No clear scaling law can be found for the dependence of the threshold of $\chi$ on $f$.
The behavior of farther layers depends crucially on the strength of the short-range attraction, $\chi$. For low $\chi$ values, the monomer concentration in the farther layers continues to decay, and the conditions are insufficient for multilayer formation. This situation is shown in Fig. 4. In the opposite case of large enough $\chi$ values (depicted in Fig. 3), the increase of the layer width causes the adsorbing polymers to interact with more than one adsorbed layer. The complexation between adjacent layers becomes stronger, allowing the monomer concentration in the adsorbing layer to increase beyond that of previous layers (see intermediate region of growth, layers 6-10 in Fig. 3). When the monomer concentration in the adsorbing layer becomes high enough (layers 10 and above in Fig. 3), the SR attraction between the different PE chains is balanced by the third virial term of the excluded volume, and the adsorbed amount in each additional layer stabilizes. This stable multilayer is characteristic of the distal region, and persists to dozens and even hundreds of layers without any noticeable decay. We note that the specific built-up of the first dozen layers is a direct consequence of our simple model of attractive interactions. A more elaborate model may be needed for quantitative comparison with experimental findings.

We now turn to the $\chi$ threshold value needed to obtain a stable multilayer formation. The threshold comes about because of the competition between the SR and electrostatic interactions. The decrease of the $\chi$ threshold with salt, as seen in Fig. 6, can be explained in the following way. Added salt screens the electrostatic interactions and results in an increase in the PE adsorbed amount and layer width 11. The thicker layers have a larger contribution to the attractive SR term in Eq. [4 and lead to a lower $\chi$ threshold.

The dependence of the threshold $\chi$ on $f$ (Fig. 7) is explained qualitatively as follows. An increase in $f$ causes an increase in the monomer-monomer repulsion between the adsorbing PE chains, and an increase in the electrostatic attraction of the adsorbing $\mathrm{PE}$ chains to the already adsorbed oppositely charged layer. The increase in the threshold $\chi$ with $f$ shows that the main effect of increasing $f$ is to decrease the adsorption, meaning that the main driving force of multilayer formation is not the charge reversal caused by the adsorbing polymer layers, but rather the SR interaction. It is important to note that in experiment [40] a threshold $f$ value for the multilayer formation was found. Above this threshold, the multilayer concentration decreases with $f$, which is in agreement with our findings. A threshold in the $f$ value is not found in our calculations, mainly because our SR interactions are externally imposed and do not depend on the value of $f$. However, the threshold can be understood qualitatively as the value of $f$ for which the polymer chains begin to interpenetrate, giving rise to the SR attraction between them. We believe that further studies are needed to better understand the origin of this threshold value.

Our simple model is subject to several limitations. First, we use the mean-field theory and the ground-state dominance approximation, valid for long PE chains. The 
adsorption of short polyelectrolyte chains requires other treatments such as molecular dynamics, Monte Carlo simulations or lattice models. Second, during the adsorption of each layer, we assume that all preceding layers are frozen, meaning that they do not dissolve back into the solution or change their spatial conformation. This assumption stems from the fact that within mean-field theory there is no way to distinguish between an adsorbed chain and a chain that is merely 'stuck' at the surface vicinity. This deficiency of mean-field does not allow us to give an accurate model for the washing procedure. Experimentally, we expect the polymer concentration to decrease, especially during the washing step, as was modeled by other techniques 52. However, due to long relaxation times we do not think that the washing will affect drastically the structure of the already adsorbed multilayer stack. Finally, we use a very simple model for the SR attraction, and do not offer any explanation for its dependence on the PE parameters. Despite these limitations, we believe that our model gives an insight for the multilayer formation problem.

Quite recently, we have become aware of a alternative mechanism for multilayer formation suggested by $\mathrm{Q}$. Wang [55. This involves multilayer formation for poor solvent condition (balanced by electrostatics). The multilayer formation can be achieved even if the polyanion and polycation chains repel each other at short distances, because the poor solvent condition induces stable multilayer builtup. The solvent condition in Wang's model plays a similar role as the attractive interaction between the cationic and anionic chains in our model.

\section{Conclusions}

We present a model aiming to explain PE multilayer formation for marginally good solvents. The model is based on strong enough short-range interactions between the polyanion and polycation. This strong short-range interaction is shown to be indispensable for our modeling of such stable multilayers. We show that the multilayers form easily in high ionic strength conditions, and that their formation does not rely exclusively on the electrostatic attraction to the previously adsorbed layers. We also calculate what is the threshold strength of the short-range interactions needed for the formation of multilayers as a function of the salinity as well as monomer charge fraction.

In our model the multilayers are quite thick and interpenetrating, while in the experiments the layers are thinner. However, we believe that this simple model gives good insight on the problem of multilayer formation, and can serve as a starting point for more refined models. A possible extension will be to use a more specific model for the short-range interactions between the PE chains, which may give a better explanation to the experimentally observed multilayer formation.

The authors would like to thank Alexander Grosberg, Jacob Klein, Henri Orland and Qiang (David) Wang for benefiting discussions. Support from the Israel Science Foundation (ISF) under grant no. 160/05 and the US-Israel Binational Foundation (BSF) under grant no. $287 / 02$ is gratefully acknowledged.

\section{References}

1. F.W. Wiegel, J. Phys A: Math. Gen. 10, 299 (1977).

2. M. Muthukumar, J. Chem. Phys. 86, 7230 (1987).

3. X. Chatellier and J.F. Joanny, J. Phys. II (France) 6, 1669 (1996).

4. R. Varoqui, A. Johner, A. Elaissari, J. Chem. Phys. 94, 6873 (1991).

5. R. Varoqui, J. Phys. II (France) 3, 1097 (1993).

6. J.F. Joanny, Eur. Phys. J. B. 9, 117 (1999).

7. I. Borukhov, D. Andelman, H. Orland, Macromolecules 31, 1665 (1998); Europhys. Lett. 32, 499 (1995).

8. I. Borukhov, D. Andelman, H. Orland, Eur. Phys. J. B 5, 869 (1998).

9. I. Borukhov, D. Andelman, H. Orland, J. Phys. Chem. B 103, 5042 (1999).

10. A. Shafir, D. Andelman, R.R. Netz, J. Chem. Phys. 119, 2355 (2003).

11. A. Shafir, D. Andelman, Phys. Rev. E. 70, 061804 (2004).

12. M. Manghi and M. Aubouy, cond-mat/0202045 preprint.

13. O.V. Borisov, E.B. Zhulina, T.M. Birshtein, J. Phys. II (France) 4, 913 (1994).

14. R.R. Netz and J.F. Joanny, Macromolecules 32, 9013 (1999).

15. A.V. Dobrynin, A. Deshkovski, M. Rubinstein, Macromolecules 34, 3421 (2001).

16. R.R. Netz and D. Andelman, in Encyclopedia of Electrochemistry, Eds. M. Urbakh and E. Giladi, Vol. I, (WileyVCH, Weinheim, 2002).

17. R.R. Netz and D. Andelman, Phys. Rep. 380, 1 (2003).

18. H.A. van der Schee and J. Lyklema, J. Phys. Chem. 88, 6661 (1984).

19. O.A. Evers, G.J. Fleer, J.M.H.M. Scheutjens, J. Lyklema, J. Coll. Interface Sci. 111, 446 (1986).

20. H.G.M. van de Steeg, M.A. Cohen Stuart, A. de Keizer, B.H. Bijsterbosch, Langmuir 8, 2538 (1992).

21. M.R. Böhmer, O.A. Evers, J.M.H.M. Scheutjens, Macromolecules 23, 2288 (1990).

22. V. Yamakov, A. Milchev, O. Borisov, B. Dünweg, J. Phys: Condens. Matter 11, 9907 (1999).

23. R. Messina, E. González-Tovar, M. Lozada-Cassou, C. Holm Europhys. Lett. 60, 383 (2002).

24. M. Ellis, C.Y. Kong, M. Muthukumar, J. Chem. Phys. 112, 8723 (2000).

25. J. McNamara, C.Y. Kong, M. Muthukumar, J. Chem. Phys. 117, 5354 (2002).

26. G. Decher, J.B. Schlenoff, Multilayer Thin Films (WileyVCH, Weinheim 2002).

27. Yu. Lvov, H. Haas, G. Decher, H. Möwald, A. Kalachev, J. Phys. Chem 97, 12835 (1993).

28. Yu. Lvov, G. Decher, H. Haas, H. Möhwald, A. Kalachev, Physica B 198, 89 (1994).

29. B.D. Jung, J.D. Hong, A. Voigt, S. Leporatti, L. Dähne, E. Donath, H. Möhwald, Coll. Surf. A: Phys. and Eng. 198-200, 483 (2002).

30. I.L. Radtchenko, G.B. Sukhorukov, H. Möwald, Coll. Surf. A: Phys. and Eng. 202, 127 (2002). 
31. R. Heuberger, G. Sukhorukov, J. Vörös, M. Textor, H. Möwald, Adv. Funct. Mater. 15, 357 (2005).

32. G. Decher, Science 277, 1232 (1997).

33. E. Huebsch, V. Ball, B. Senger, G. Decher, J.-C. Voegel, P. Schaaf, Langmuir 20, 1980 (2004).

34. J. Schmitt, T. Grünwald, G. Decher, P. S. Pershan, K. Kjaer, M. Lösche, Macromolecules 26, 7058 (1993).

35. M. Lösche, J. Schmitt, G. Decher, W. G. Bouwman, K. Kjaer, Macromolecules 31, 8893 (1998).

36. P. Scheutz, F. Caruso, Adv. Funct. Mater. 13, 929 (2003).

37. P. Schuetz, F. Caruso, Coll. Surf. A: Phys. and Eng 207, 33 (2002).

38. E. Poptoshev, B. Schoeler, F. Caruso, Langmuir 20, 829 (2004).

39. U. Voigt, V. Khrenov, K. Tauer, M. Halm, W. Jaeger, R. von Klitzing, J. Phys.: Condens. Matter 15, S213 (2003).

40. U. Voigt, W. Jaeger, G. Findenegg, R. von Klitzing, J. Phys. Chem B 107, 5273 (2003).

41. R. Steitz, V. Leiner, R. Siebrecht, R. v. Kliting, Coll. Surf. A.: Phys. and Eng. 163, 63 (2000).

42. R. v. Klitzing, J. E. Wong, W. Jaeger, R. Steitz, Curr. Opin. Coll. Int. Sci. 9, 158 (2004).

43. Z. Adamczyk, M. Zembala, P. Warszynski, B. Jachimska, Langmuir 20, 10517 (2004).

44. E. Blomberg, E. Poptoshev, P.M. Claesson, F. Caruso, Langmuir 20, 5432 (2004).

45. J. Cho, J.F. Quinn, F. Caruso, J. Am. Chem. Soc. 126, $2270(2004)$.

46. G. Greene, R. Tannenbaum, App. Surf. Sci. 233, 336 (2004).

47. G. Greene, G. Yau, R. Tannenbaum, Langmuir 20, 2739 (2004).

48. M. Castelnovo, J.F. Joanny, Langmuir 16, 7524 (2000).

49. S.Y. Park, M.F. Rubner, A.M. Mayes, Langmuir 18, 9600 (2002)

50. R. Messina, Macromolecules 37, 621 (2004).

51. Q. Wang, Macromolecules 38, 8911 (2005).

52. P.A. Patel, J. Jeon, P.T. Marher, A.V. Dobrynin, Langmuir 21, 6113 (2005).

53. P.G. de Gennes, Macromolecules 14, 1637 (1981).

54. W.H. Press, B.P. Flannery, S.A. Teukolsky, W.T. Vetterling, Numerical Recipes in C: The Art of Scientific Computing, (Cambridge University, Cambridge 1992), Chap. 17, p. 762 .

55. Q. Wang, cond-mat 0507118 (submitted) . 